Noname manuscript No.

(will be inserted by the editor)

\title{
The Tracking Analysis in the Q-weak Experiment
}

J. Pan for the Q-weak Collaboration:

D. Androic · D. S. Armstrong • A.

Asaturyan - T. Averett - J. Balewski .

J. Beaufait - R. S. Beminiwattha • J.

Benesch • F. Benmokhtar • J. Birchall •

R. D. Carlini · G. D. Cates • J. C.

Cornejo - S. Covrig - M. M. Dalton • C.

A. Davis - W. Deconinck • J. Diefenbach •

J. F. Dowd · J. A. Dunne - D. Dutta .

W. S. Duvall • M. Elaasar • W. R. Falk •

J. M. Finn - T. Forest • D. Gaskell · M.

T. W. Gericke · J. Grames • V. M. Gray •

K. Grimm • F. Guo • J. R. Hoskins • K.

Johnston - D. Jones • M. Jones • R.

Jones - M. Kargiantoulakis - P. M. King •

E. Korkmaz • S. Kowalski • J. Leacock .

J. Leckey • A. R. Lee • J. H. Lee · L.

Lee - S. MacEwan • D. Mack • J. A.

Magee - R. Mahurin • J. Mammei • J. W.

Martin - M. J. McHugh • D. Meekins • J.

Mei • R. Michaels • A. Micherdzinska •

A. Mkrtchyan - H. Mkrtchyan - N.

Morgan - K. E. Myers • A. Narayan • L.

Z. Ndukum • V. Nelyubin • Nuruzzaman •

W.T.H van Oers • A. K. Opper • S. A.

Page • J. Pan • K. D. Paschke - S. K.

Phillips • M. L. Pitt • M. Poelker •

J. F. Rajotte - W. D. Ramsay • J.

Roche - B. Sawatzky - T. Seva • M. H.

Shabestari - R. Silwal • N. Simicevic • G.

R. Smith - P. Solvignon - D. T. Spayde •

A. Subedi - R. Subedi - R. Suleiman - V. Tadevosyan - W. A. Tobias - V. Tvaskis . B. Waidyawansa - P. Wang - S. P. Wells . S. A. Wood · S. Yang • R. D. Young • S. Zhamkochyan

Received: date / Accepted: date

J. Pan

University of Manitoba

Tel.: +1-204-474-7910

E-mail: jpan@jlab.org 
Abstract The Q-weak experiment at Jefferson Laboratory measured the parity violating asymmetry $\left(A_{P V}\right)$ in elastic electron-proton scattering at small momentum transfer squared $\left(Q^{2}=0.025(\mathrm{GeV} / \mathrm{c})^{2}\right)$, with the aim of extracting the proton's weak charge $\left(Q_{W}^{p}\right)$ to an accuracy of $5 \%$. As one of the major uncertainty contribution sources to $Q_{W}^{p}, Q^{2}$ needs to be determined to $\sim 1 \%$ so as to reach the proposed experimental precision. For this purpose, two sets of high resolution tracking chambers were employed in the experiment, to measure tracks before and after the magnetic spectrometer. Data collected by the tracking system were then reconstructed with dedicated software into individual electron trajectories for experimental kinematics determination. The Q-weak kinematics and the analysis scheme for tracking data are briefly described here. The sources that contribute to the uncertainty of $Q^{2}$ are discussed, and the current analysis status is reported.

Keywords Parity violating asymmetry · Momentum transfer · Kinematics

PACS 14.20.Dh $\cdot$ 13.60.Fz $\cdot 29.40 . \mathrm{Gx} \cdot 29.85 . \mathrm{Fj} \cdot 29.85 . \mathrm{Ca}$

\section{Introduction}

The Q-weak experiment at Jefferson Laboratory [1] measured the parity violating asymmetry $\left(A_{P V}\right)$ in elastic electron-proton scattering at small 4-momentum transfer squared $\left(Q^{2}=0.025(\mathrm{GeV} / \mathrm{c})^{2}\right)$, with the aim of extracting the proton's weak charge $\left(Q_{W}^{p}=1-4 \sin ^{2} \theta_{W} \sim 0.072\right.$ at tree level) to high precision via [2]:

$$
A_{P V}=\frac{-G_{F} Q^{2}}{4 \pi \alpha \sqrt{2}}\left[Q_{W}^{p}+Q^{2} B\left(Q^{2}, \theta\right)\right] .
$$

In Eqn. $1, G_{F}$ is the Fermi constant, $\alpha$ is the fine structure constant, $\theta$ is the laboratory electron scattering angle, and $B\left(Q^{2}, \theta\right)$ is a hadronic structure term which can be extracted from the existing PVES data $\left(\sim 30 \%\right.$ contribution to $\left.A_{P V}\right)$. Since the proton's weak charge relates to the measured asymmetry by a factor which is proportional to $Q^{2}$, the uncertainty of $Q^{2}$ contributes directly to that of $Q_{W}^{p}$. The experimental goal is to extract $Q_{W}^{p}$ to approximately $5 \%$. To reach the proposed experimental precision, $Q^{2}$ needs to be determined to $\sim 1 \%$ if a $\sim 8 \mathrm{ppb}$ precision of $A_{P V}(\sim-300 \mathrm{ppb})$ measuremen could be achieved. For elastic electron-proton scattering, 4-momentum transfer squared is given by:

$$
Q^{2}=2 E^{2} \frac{1-\cos \theta}{1+\frac{E}{M_{p}}(1-\cos \theta)},
$$

where $E$ is the energy of incident electron and $M_{p}$ is the rest mass of a proton. By knowing the laboratory scattering angle $\theta$ and incident beam energy $E, Q^{2}$ can be determined. The $Q^{2}$ determination is conducted via the $\mathrm{Q}$-weak tracking system and analysis.

\section{Experiment Setup and Operation}

Q-weak employed a $1.165 \mathrm{GeV}, 89 \%$ longitudinally polarized electron beam colliding with a $35 \mathrm{~cm}$ long liquid hydrogen target (see Fig. 1) [3]. The scattered 


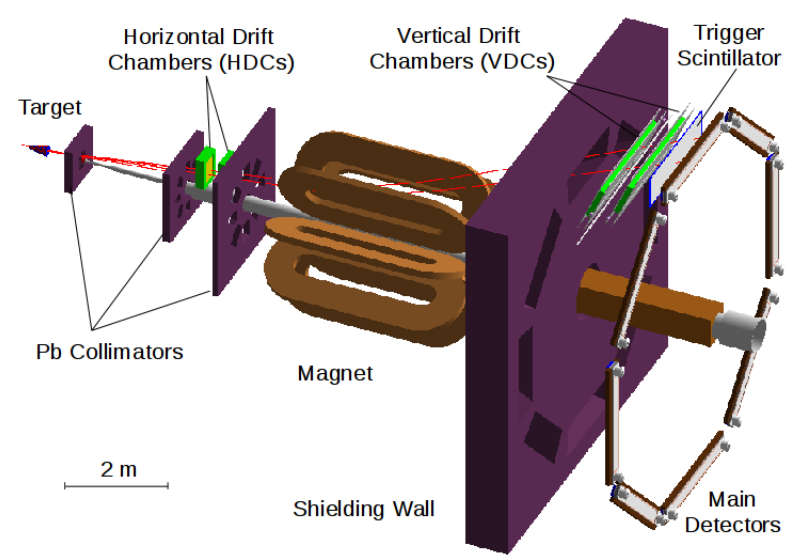

Fig. 1 Q-weak experimental apparatus with simulated electron trajectories (trigger scintillator, horizontal and vertical drift chambers were used in low current tracking mode only).

electrons with scattering angle of $\sim 8^{\circ}$ were selected by a set of $\mathrm{Pb}$ collimators, momentum-analyzed by a resistive 8-fold toroidal magnetic spectrometer (QTOR), and detected by the main $\check{C}$ erenkov detectors made of fused silica.

Two sets of tracking chambers, horizontal drift chambers (HDCs, 12 planes, $200 \mu \mathrm{m}$ resolution) and vertical drift chambers (VDCs, 4 planes, $300 \mu \mathrm{m}$ resolution), were employed to measure tracks before and after the magnetic spectrometer [3]. The HDC data are used to determine the scattered electron trajectories upstream of the QTOR magnet; these are tracked back to the target to establish the interaction vertex and the scattering angle $\theta$ required for determining the $Q^{2}$ distribution. The VDC data are used in conjunction with the tracks from the HDCs and the known magnetic field of QTOR to determine the scattered momentum and thereby identify elastically scattered electrons.

For high precision asymmetry measurements, Q-weak was operated at high beam current $(\sim 180 \mu \mathrm{A})$ with a luminosity of $2 \times 10^{39} \mathrm{~s}^{-1} \mathrm{~cm}^{-2}$ for one acceleratoryear. Dedicated low-current $(\sim 50 \mathrm{pA})$ tracking runs were periodically conducted for $Q^{2}$ determination with tracking detectors.

\section{Tracking Analysis}

Data collected by the tracking system were reconstructed with dedicated software (patterned on the HERMES algorithm [4]) following the procedure below:

- Assemble all hit information from data stream and identify hit locations in each wire plane based on wire number and drift time,

- Sort hits into track segments in HDCs and VDCs by applying pattern recognition and least squares fits,

- Bridge track segments before and after magnet into a complete track using the 4th-order Runge Kutta [5] trajectory integration in the magnetic field,

- Extract track parameters for kinematics determination.

Note that the physical $Q^{2}$ at the scattering vertex is not directly observable, as the interaction happens inside the $35 \mathrm{~cm}$ long liquid hydrogen target. In or- 


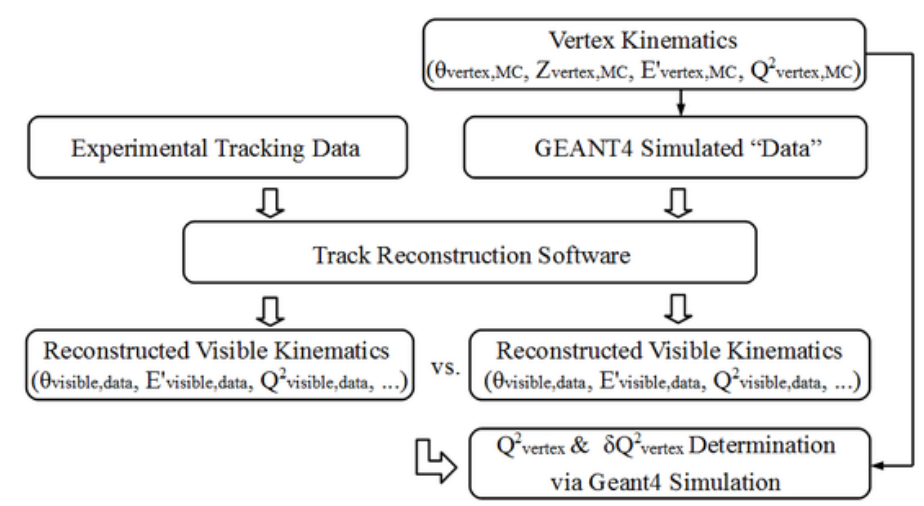

Fig. 2 Tracking analysis flowchart

der to deduce the interaction vertex kinematics from observed track parameters, Monte Carlo simulation is required. Simulated tracks with known kinematics are fed through the same tracking analysis software as used to reconstruct tracks for the experimental data. By comparing the visible kinematics determined by tracking data against simulated data, the visible kinematics are verified, and the final vertex kinematics and uncertainties can be extracted from the Monte Carlo simulation (see Fig. 2 for a tracking analysis flowchart). The average $Q^{2}$ obtained from tracking analysis is weighted by the light response of the main detectors for use in determining the proton's weak charge via Eqn. 1.

The overall $Q^{2}$ uncertainty is limited by the accuracy to which we can simulate the observed experimental conditions. Although the statistical uncertainty is negligible, there are many other sources which contribute to the $Q^{2}$ uncertainty. The most significant ones are beam and target related, including beam raster, position, angle and energy, electron energy loss in the liquid hydrogen target, target location and the effect of Aluminum target windows on the observed $Q^{2}$. Detectors and spectrometer related sources also make large contributions, such as the detector geometry and inefficiency, collimator geometry, absolute field values of the magnetic spectrometer, rate dependent effects, run-to-run stability and octant dependency.

In addition, analysis software parameters and data cuts, and data to simulation comparisons are considerable sources of uncertainty. To quantify their contributions, the sensitivity of $Q^{2}$ to different uncertainty sources is being studied by using GEANT4 Monte Carlo simulations and comparisons with experimental tracking data $^{1}$. For example, the target Z-position is one of the highest nails on the uncertainty table. Fig. 3 shows an example of how $Q^{2}$ varies with target Z-position. The sensitivity of $Q^{2}$ is determined by the slope of the line. With further improvements to parameters of the tracking chamber geometry, we will be able to quantify the uncertainty from chamber-to-chamber alignment, and obtain an independent target Z-position from tracking data alone, which essentially could be used to verify the survey data for the target location.

1 For a tracking simulation with an equivalent statistical uncertainty of 1 second experimental data at $50 \mathrm{pA}$ beam current, the computing time is $\sim 1$ hour on a $2 \mathrm{GHz} \mathrm{CPU}$. 


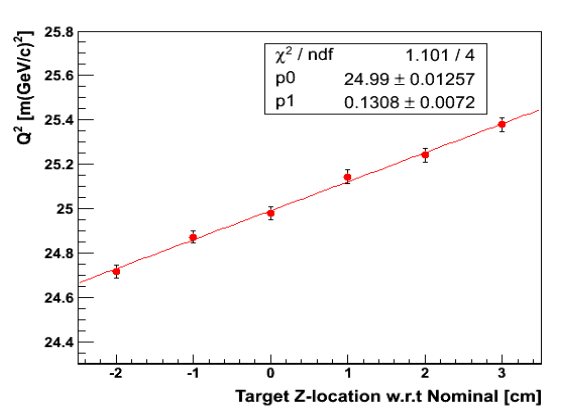

Fig. 3 Sensitivity of $Q^{2}$ to target Z-location

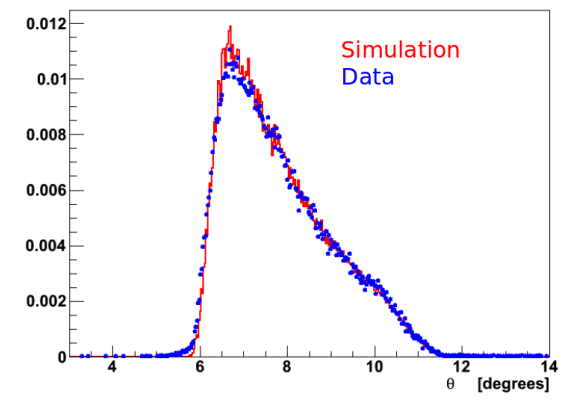

Fig. 4 Simulation to data comparison: electron scattering angle

\section{Status and Outlook}

A subset of the Q-weak commissioning data was published in 2013, yielding the first experimental determination of the proton's weak charge. For this relatively small data sample, the $\left\langle Q^{2}\right\rangle$ was determined to a precision of $2.4 \%$, and was not a major contribution to the error in $Q_{W}^{p}$. The full analysis of Qweak Run 1 and Run 2 physics data $(\sim 25 \times$ more than published previously) and ancillary measurements is nearing completion now, with current efforts focussing on quantifying the systematic uncertainties. The Q-weak tracking analysis is also in its final stage - completing the $Q^{2}$ uncertainty table, after multiple passes of data replay and extensive simulations. For the overall effort of pursuing the greatest new physics reach via the smallest achievable total error bar, attention of tracking analysis is now focussed on identifying the sources of systematic uncertainties and estimating their contributions to the total $Q^{2}$ uncertainty. Some preliminary data to simulation comparison studies were also carried out and initial uncertainties were estimated. For example, the scattering angle $\theta$ determination is one of the dominant uncertainties in $Q^{2}$. Fig. 4 shows a comparison of the simulated scattering angle to that measured with tracking data. At this stage, data agree with simulation to better than $0.5 \%$. Continuous effort on the tracking analysis will focus on further improvements to the tracking algorithms and detector simulations needed to pin down any remaining contributions to the $Q^{2}$ uncertainty.

Acknowledgements The author would like to acknowledge Drs. S. A. Page, D. S. Armstrong, M. L. Pitt and other fellow Q-weak Collaborators. This work is supported by NSERC and DOE.

\section{References}

1. D. Androic et al. (Q-weak Collaboration), First Determination of the Weak Charge of the Proton, Phys. Rev. Lett. 111, 141803 (2013)

2. Young, R.D., et al., Testing the Standard Model by Precision Measurement of the Weak Charges of Quarks, Phys. Rev. Lett. 99, 122003 (2007)

3. Allison, T., et al. (Q-weak Collaboration), The Q-weak Experimental Apparatus, Nucl. Instrum. Meth. A781, 105-133 (2015)

4. Ackerstaff, K., et. al. (HERMES), Nucl. Instrum. Meth. A417, 230 (1998)

5. Press, W.H., et al., Numerical Recipes in C: The Art of Scientific Computing, Second Edition Cambridge University Press (1993) 